\title{
Aplanamiento de las cabezas de los metacarpianos
}

\author{
García Cañas R. ${ }^{1}$, Muñoz Ruiz A. ${ }^{2}$, Sopesén Veramendi JL. ${ }^{3}$
}

Sanid. mil. 2015; 71 (1): 57-58; ISSN: 1887-8571

\section{DESCRIPCIÓN DEL CASO}

Paciente varón de 42 años sin alergias conocidas y con antecedentes personales de desprendimiento de retina bilateral y paladar hendido. Valorado en consultas de Traumatología por traumatismo casual sobre dorso de mano derecha. En la exploración se observa eritema y tumefacción en dorso de mano a nivel de cabeza de $4^{\circ}$ metacarpiano, dolor leve a la palpación de estructuras óseas y una marcada limitación para la flexión

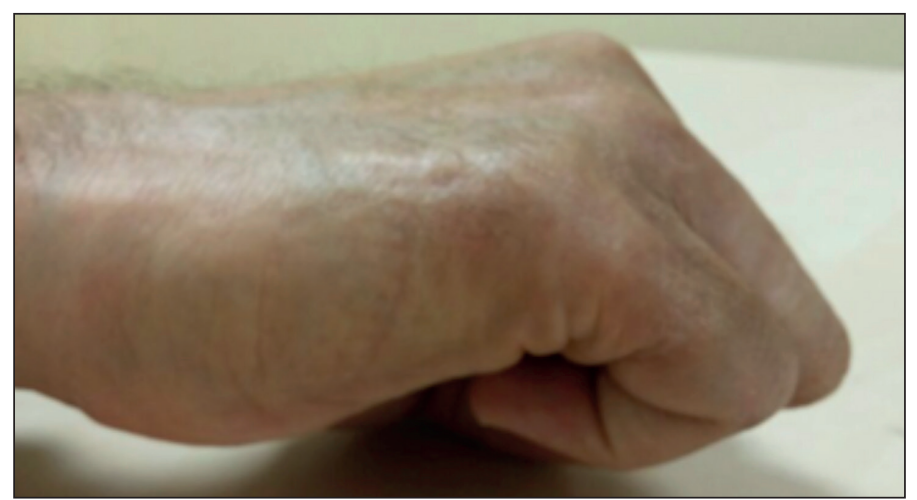

Figura 1. Imagen de la mano derecha.

completa de la articulación metacarpofalángica de $3^{\circ}$ y $4^{\circ}$ dedos (Figura 1). El paciente manifiesta que dicha limitación es anterior al traumatismo en varios años. Presenta similar limitación en la mano contralateral que afecta a los mismos dedos, $3^{\circ}$ y $4^{\circ}$.

Se solicita una radiografía simple de ambas manos (Figuras 2 y 3), donde se objetiva la ausencia de lesión traumática ósea en zona de contusión, y como hallazgo radiológico, la presencia de aplanamiento de las cabezas metacarpianas $3^{\mathrm{a}}$ y $4^{\mathrm{a}}$ en ambas manos, con lesión espejo en la base de sus falanges correspondientes, lo que da un aspecto «cuadrado» a la articulación.

Se pauta tratamiento para la contusión (reposo, crioterapia y toma de AINEs) y se le cita en dos semanas para una exploración funcional no alterada por el dolor.

${ }^{1}$ Tte. Médico. EMISAN.

${ }^{2}$ Cte. Médico. Excedencia.

${ }^{3}$ Cte. Médico.

Servicio de Cirugía Ortopédica y Traumatología. Hospital Central de la Defensa Gómez Ulla. Madrid. España.

Dirección para correspondencia: Rafael García Cañas. Servicio de Cirugía Ortopédica y Traumatología. Hospital Central de la Defensa Gómez Ulla. Glorieta del Ejército 1. 28047 Madrid. España.rgarc18@oc.mde.es

Recibido: 2 de diciembre de 2014

Aceptado: 20 de enero de 2015

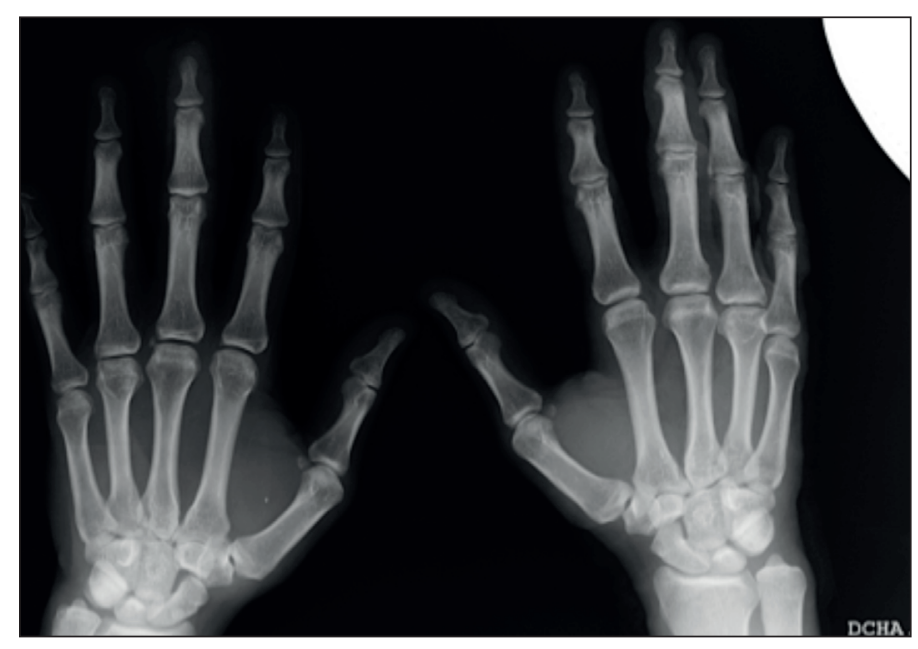

Figura 2. Rx simple de ambas manos.

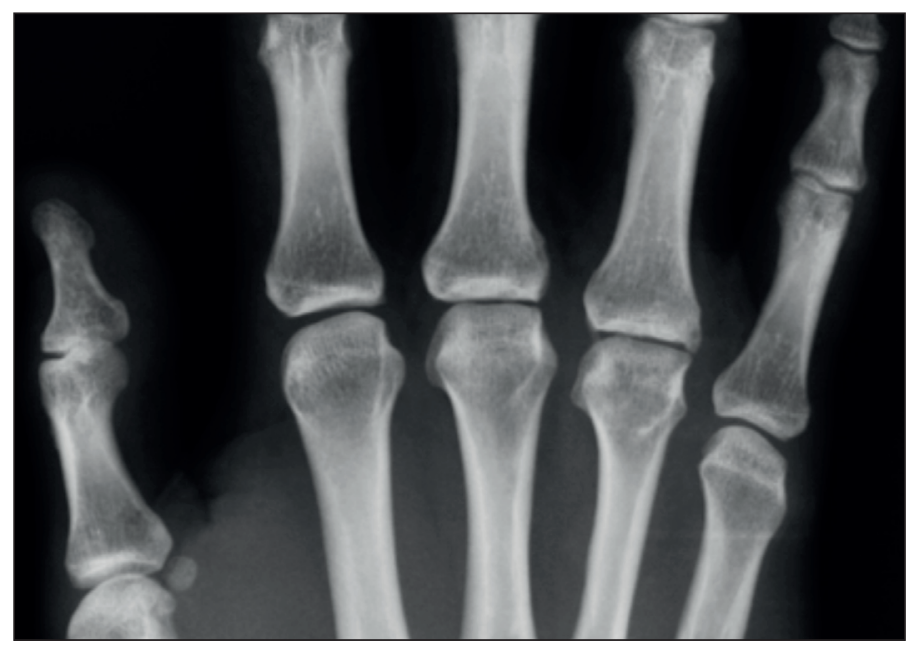

Figura 3. Detalle de la Rx de mano derecha.

A las dos semanas, el paciente refiere mejoría completa de su proceso traumático. Se observa disminución del arco de movilidad metacarpofalángico en ambas manos (valores normales: extensión $0-20^{\circ}$ y flexión $0-90^{\circ}$ ) consistente en pérdida en la mano derecha de los últimos $30^{\circ}$ de flexión en el $3^{\circ}$ dedo y de los últimos $25^{\circ}$ en el $4^{\circ}$. En la mano izquierda tan solo se aprecia la pérdida de los últimos $20^{\circ}$ en la flexión del $3^{\circ}$ y $4^{\circ}$ dedos (coincidente con una menor afectación radiológica en esa mano). Pese a la afectación radiológica de más articulaciones, no objetivamos déficit de movilidad en ninguna otra.

No tiene dolor actualmente, ni lo ha sufrido en el pasado, siendo su queja principal la limitación que presenta en actividades que requieren formar un puño con la mano. 


\section{Diagnóstico: enfermedad de dieterich}

\section{DISCUSIÓN}

La necrosis avascular de la cabeza de los metacarpianos conocida como «Enfermedad de Dieterich» es una patología muy infrecuente. Desde que en 1932 fuera descrita por H. Dieterich ${ }^{1}$, no más de 60 casos han sido descritos en la literatura ${ }^{2}$. La patogenia de esta particular lesión, al igual que el resto de necrosis de otras localizaciones no está bien definida. Existen varios factores desencadenantes descritos, tales como: los traumatismos, la toma de corticoides, las malformaciones congénitas y/o la asociación con enfermedades reumáticas.

El tercer dedo es el más frecuentemente afectado en este proceso $^{1}$, aunque también se han descrito casos en las demás cabezas metacarpianas. La clínica típica es una tríada sintomática que consta de: dolor, inflamación de la articulación metacapofalángica y limitación funcional. El diagnóstico se basa en el estudio radiográfico, si bien en los estadios iniciales suele ser inespecífico, más adelante se presenta como una disminución de la interlínea articular junto con la presencia de colapso subcondral en la cabeza metacarpiana ${ }^{3}$. En caso de dudas en el diagnóstico, la gammagrafía ósea y la resonancia magnética pueden ser de utilidad.

El tratamiento dependerá del estadio evolutivo de la enfermedad. En las fases iniciales se recomienda el tratamiento conservador basado en reposo y la toma de AINEs ${ }^{4}$. No obstante, creemos que en estos estadios iniciales, las técnicas de mosaicoplastia con aloinjerto y el implante de condrocitos autólogos, podrían tener un papel fundamental como alternativa a técnicas quirúrgicas más agresivas ${ }^{5,6}$.

En cambio, el tratamiento de la necrosis en los estadios más avanzados dependerá del grado de degeneración articular. Así, se puede realizar una osteotomía flexora de la cabeza metacarpiana $^{7,8}$, si sólo existe colapso subcondral, mientras que si existen signos artrósicos evidentes, el tratamiento de elección es la artroplastia ${ }^{9}$.

\section{BIBLIOGRAFÍA}

1. Dieterich H. Die subchondrale Herderkrankung am Metacarpale III. Arch Klin Chir 1932;171:555-567. (Original no consultado, citado por: Proubasta I.R., Lamas C.G., Itarte J.P., Pérez F.M. Enfermedad de Dieterich. Necrosis avascular idiopática de la cabeza metacarpiana. AVANCES 2003; 33: 195-8.)

2. Karlakki S.L., Bindra R.R. Idiopathic avascular necrosis of the metacarpal head. Clin Orthop Relat Res. 2003 Jan;(406):103-8.

3. Barnes N.A., Howes A.J., Jeffers H., Carty H. Quiz case. Avascular necrosis of the metacarpal head III. Eur J Radiol. 2000 Nov;36(2):115-7.

4. Kalenderer O, Ağuş H, Ozlük S. Avascular necrosis of the third metacarpal head: a case report. Acta Orthop Traumatol Turc 2004;38:154-156.

5. Braun B.J., Brandenburg L.O., Braun C. Treatment of a partial avascular necrosis of a metacarpal head (Morbus Mauclaire Dieterich's Disease) utilizing the ostechondral autograft transfer system (OATS) technique. Handchir Mikrochir Plast Chir. 2012 Jan;44(1):35-9.

6. Maes M., Hansen L., Cheyns P. Osteochondral mosaicplasty as a treatment method for bilateral avascular necrosis of the long finger metacarpal: case report. J Hand Surg Am. 2010;35(8):1264-8

7. Wada M, Toh S, Iwaya D, Harata S. Flexion osteotomy of the metacarpal neck: a treatment method for avascular necrosis of the head of the third metacarpal: a case report. J Bone Joint Surg Am. 2002 Feb;84-A(2):274-6.

8. Ohta S., Kakinoki R, Fujita S, Noguchi T. Open wedge flexion osteotomy of the metacarpal neck for the avascular necrosis of the third metacarpal head: case report. Hand Surg. 2012;17(2):251-3.

9. Proubasta I.R., Lamas C.G., Itarte J.P., Pérez F.M. Enfermedad de Dieterich. Necrosis avascular idiopática de la cabeza metacarpiana. AVANCES 2003; 33: 195-8 